\title{
MUCORMYCOSE RHINOSINUSIENNE A EXTENSION PALATINE
}

\author{
Z. MZIOU, R. MOATEMRI, S. TOUMI *, S. AYACHI, M. OMEZZINE, M. CHELBI, A. SLAMA, \\ CH. LAOUANI**, H. KHOCHTALI
}

\author{
SERVICE DE STOMATOLOGIE ET DE CHIRURGIE MAXILLO-FACIALE \\ SERVICE DE MÉDECINE INTERNE \\ CHU SAHLOUL SOUSSE. TUNISIE
}

\begin{abstract}
Les mucormycoses sont des infections fongiques, aigues, rares et souvent fatales.
Elles touchent avec prédilection les sujets immunodéprimés. La forme rhinocérébrale est la plus fréquente. Le diagnostic repose sur l'examen clinique, anatomopathologique et mycologique. L'approche thérapeutique doit être multidisciplinaire.

Les auteurs rapportent l'histoire clinique d'un patient, ayant présenté une mucormycose rhinosinusienne avec atteinte du palais au décours d'une infection dentaire. A travers cette observation, ils discutent les différents aspects cliniques, les moyens du diagnostic et les modalités thérapeutiques de la mucormycose rhinocérébrale.

Mots clés : Infection fongique, mucormycose rhinocérébrale, zygomycètes, pronostic, traitement.
\end{abstract}

\section{SUMMARY}

Mucormycosis is a rare and acute fungal infection, which is frequently lethal. It's usually observed in immunodepressed patients. Rhinocerebral mucormycosis is the most frequent of mucormycosis locations. The diagnosis rests on the clinical, mycological and anatomopathological examination. The therapeutic approach must be multidisciplinary.

The authors report a clinical history of patient, having presented a rhino maxillary palatal mucormycosis probably caused by a dental infection. This observation provides on opportunity to recall clinical, histopathological, and therapeutic aspects of rhinocerebral mucormycosis.

Keywords : Fungal infection, rhinocerebral mucormycosis, zygomycetes, prognosis, treatment

\section{INTRODUCTION}

La mucormycose est une affection fongique, opportuniste, rapidement extensive, de diagnostic souvent tardif, responsable d'une morbidité et mortalité élevée. Elle survient généralement sur des terrains d'immunosuppression. Les agents responsables sont des champignons appartenant à la classe des zygomètes du groupe mucorale. La forme rhino orbito cérébrale est la plus fréquente. Le diagnostic positif repose sur l'examen anatomopathologique et mycologique. L'évolution dépend de la précocité du diagnostic, de la prise en charge et de l'absence d'atteinte cérébrale.

\section{OBSERVATION}

Patient, âgé de 35 ans, sans antécédents particuliers, hospitalisé pour décompensation acido-cétosique inaugurale d'un diabète méconnu suite à une infection buccodentaire. Une semaine avant l'hospitalisation, le patient avait présenté des odontalgies de l'arcade dentaire supérieure droite traitées en ambulatoire. L'examen à l'admission trouvait une température à $38^{\circ} \mathrm{C}$ et une polypnée à 26 cycles par minute. L'examen de la cavité buccale notait plusieurs caries dentaires au niveau de l'hémi-arcade dentaire supérieure droite avec un aspect nécrosé de la muqueuse hémipalatine droite. L'examen biologique a révélé une acidose métabolique sévère et un syndrome inflammatoire. Un traitement à base d'insuline ordinaire et d'antibiotique (amoxicilline + métronidazole) était instauré.

Vingt quatre heures plus tard, on a noté l'apparition d'un placard inflammatoire para-nasal et génien haut avec une extension de la nécrose palatine droite (Fig 1).

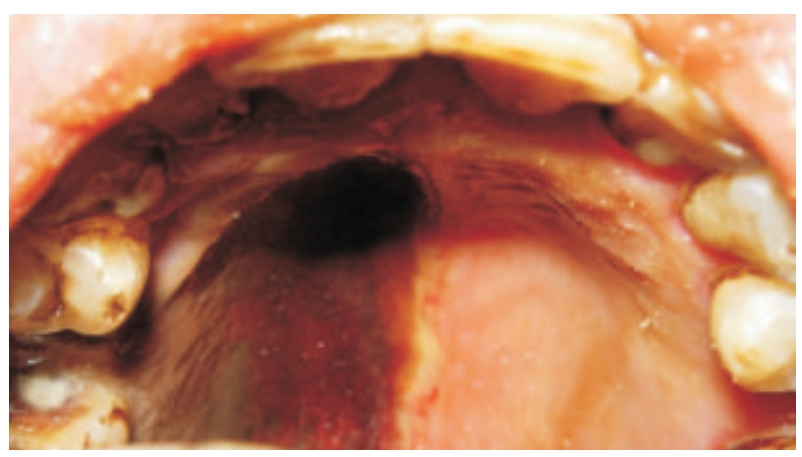

Fig. 1 : Nécrose de la fibromuqueuse hémipalatine droite.

Un scanner du massif facial a montré un processus lytique centré sur le maxillaire droit, une sinusite maxillaire homolatérale et des micro-abcès de la fosse infra temporale droite (Fig 2). 

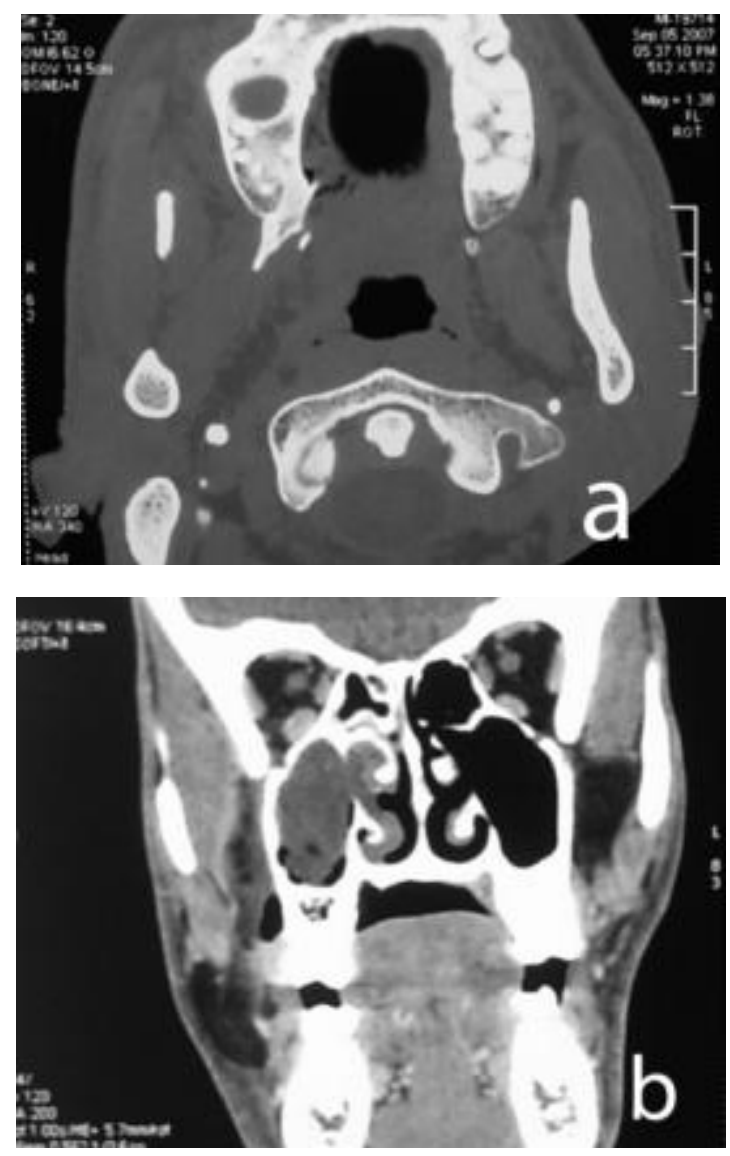

Fig. 2 : TDM du massif facial :

a- coupe axiale en fenêtre osseuse montant un processus lytique centré sur le secteur prémolo-molaire maxillaire droit. b- coupe coronale en fenêtre parenchymateuse montrant une nécrose graisseuse avec des micro-abcès au niveau de la fosse infra temporale droite associée à une opacité maxillaire droite.

Devant ce tableau clinique, une mucormycose rhino-sinusienne était suspectée. Lors de l'excision du tissu nécrosé hémipalatin droit, une thrombose du pédicule palatin postérieur a été constatée ( Fig 3).

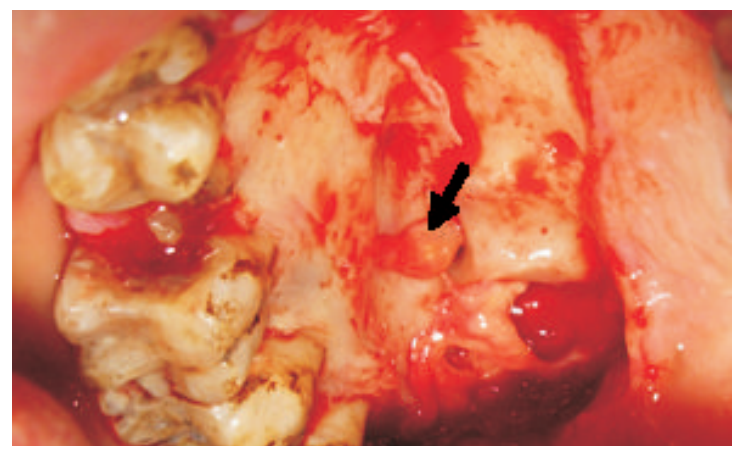

Fig. 3 : Aspect de l'hémipalais droit après débridement chirurgical montrant la thrombose de l'artère palatine postérieure
Un drainage de la cavité sinusienne a été fait par une voie de CADWELL-LUC. Un traitement par Amphotéricine B à la dose de $1 \mathrm{mg} / \mathrm{Kg} / \mathrm{J}$ était instauré, en association à une antibiothérapie ( amoxicilline et acide clavulanique, métronidazole et Gentamycine) par voie parentérale, ainsi qu'une héparinothérapie à dose curative. L'examen bactériologique des prélèvements réalisés en per opératoire avait isolé un Streptococcus B et Klebsiella pneumoniae. L'examen parasitologique notait des filaments mycéliens identifiés à Rhizopus aerhizus. Devant la persistance de l'œdème génien droit, un scanner de contrôle du massif facial a été demandé. II a montré un abcès de la loge infra temporale droite, un épaississement et une densification de la graisse endo-orbitaire homolatérale, sans extension endocrânienne. Un drainage de la fosse infra-temporale, par voie trans-sinusienne maxillaire droite a été réalisé. L'évolution favorable a permis l'arrêt du traitement antifongique au bout de 45 jours.

\section{DISCUSSION}

La mucormycose est une affection oppurtuniste, rare, consécutive à la prolifération dans les tissus d'un champignon de la famille des Zygomycètes, de la classe des mucorales (1). Les principaux germes responsables de l'infection chez l'homme sont Rhizopus, Mucor et Absidia $(1,2)$. Ces microorganismes sont ubiquitaires, ils sont omniprésents dans l'environnement sous formes de spores dans l'air, la terre, les végétaux en décomposition et les produits d'origine animale (3).

Habituellement ces microorganismes sont saprophytes chez l'homme, ils deviennent pathogènes chez les patients immunodéprimés en particulier les diabétiques surtout en décompensation acidocétosique $(1,2,4)$, ainsi que les patients atteints d'hémopathie maligne ou recevant un traitement immunosuppresseur, une corticothérapie au long cours ou une chimiothérapie, les insuffisants rénaux et les sidéens $(1,2,3,4)$.

La forme rhino-orbito-cérébrale, décrite pour la première fois en 1943 par Gregory, constitue la forme clinique la plus fréquente, elle représente près de la moitié des cas de mucormycoses chez l'homme (in 5). Cette forme a une porte d'entrée essentiellement respiratoire par l'inhalation de spores asexuées qui se fixent sur les voies nasales et les sinus (5). La porte d'entrée dentaire de la mycose est à discuter chez notre patient.

La mucormycose a pour particularité le tropisme vasculaire élevé de ses agents, qui envahissent la paroi des vaisseaux par les filaments mycéliens ce qui entraine les mucothrombosis $(1,6)$. Ces thromboses sont sources d'embole et d'obstructions vasculaires déterminant la nécrose tissulaire (5). Le diabète semble aggraver ces phénomènes de nécrose par les lésions de microangiopathies déjà présentes $(4,7,8)$.

La présentation clinique de la mucormycose rhinocérébrale est peu spécifique associant des signes nasosinu- 
siens, ophtalmologiques et neurologiques à degrés variables (2), associés à une fièvre et altération de l'état général (4). L'apparition de lésions nécrotiques sous forme d'escarres de la région orbito-nasale, du palais et du voile ou du plancher buccal attire l'attention et doit faire évoquer le diagnostic, surtout chez un terrain d'immunodépression(2,4).

L'imagerie permet de rechercher des signes d'agressivité, telle qu'une infiltration de la graisse de la fosse infra-temporale, qui représente pour plusieurs auteurs le réservoir de la mucormycose, une destruction osseuse et une extension vers l'encéphale $(5,9)$.

Le diagnostic de certitude repose sur les prélèvements biopsiques précoces et profonds et/ou sur les prélèvements mycologiques. L'examen anatomopathologique confirme le diagnostic en révélant la présence de filaments ou hyphes mycéliens, épais, courts, non septés, présentant des ramifications à angles droits portant des sporanges ou des sporocytes de formes variables selon le genre, au sein de réaction inflammatoire pyogénique $(1,2,10)$. Les microorganismes sont mis en évidence par des colorations histochimiques spéciales: PAS et GomoriGrocott (10). Seuls l'examen mycologique direct et la culture permettent de confirmer le diagnostic et d'identifier le genre et l'espèce incriminée dans l'infection. Elle permet également d'étudier la sensibilité aux antifongiques (11). Le traitement de la mucormycose est médicochirurgical reposant sur l'antifongique : «Amphotéricine $B$ » par voie intraveineuse à la dose de $1 \mathrm{mg} / \mathrm{kg} / \mathrm{jour}$, le débridement chirurgical des tissus nécrosés et l'équilibration du diabète ou de tout autre facteur de risque $(1,10,12)$.

Actuellement, l'utilisation de la forme liposomale de l'Amphotéricine B permet l'administration de hautes doses thérapeutiques afin d'améliorer le taux de réussite thérapeutique et de diminuer les effets indésirables (12, 13).
Le débridement chirurgical des lésions permet au traitement systémique d'atteindre les zones infestées, isolées par les phénomènes de thromboses vasculaires et par ailleurs, de réduire la charge fongique $(4,12,14,15)$.

Le pronostic fonctionnel et vital de cette affection est grave. En effet, le taux de mortalité reste élevé malgré les progrès thérapeutiques puisqu'il atteint 20 à $50 \%$ des cas $(1,2,4,12,15,16)$. La survie au cours de mucormycose rhinocérébrale est fonction du délai rapide de leur prise en charge. Dans la série de Yohai et al, la survie passe de $76 \%$ en cas de traitement dans un délai de moins de 7 jours à $40 \%$ en cas de traitement débutant au-delà de deux semaines du début des symptômes. (17).

\section{CONCLUSION}

La mucomycose est une affection rare, présentant une symptomatologie polymorphe et non spécifique, survenant essentiellement chez des sujets immunodéprimés. Cette infection fongique grave nécessite une approche multidisciplinaire. Le pronostic fonctionnel et vital est lié à la rapidité du diagnostic qu'il faut savoir évoquer devant des lésions non spécifiques, afin d'instaurer un traitement adapté.

\section{REFERENCES}

1- Spellberg B, Edwards Jr J, Ibrahim A. Novel perspectives on mucormycosis: pathophysiology, presentation and management. Clin Microbiol Rev. 2005; 18:556-69.

2- Glockner A, Vehreschild JJ, Cornely OA. Zygomycosis: current epidemiological aspects. Mycoses 2007; 50(1):50-5.

3- Talmi YP, Goldschmied-Reouven A., Bakon M. et al. Rhino-orbital and rhinoorbito-cerebral mucormycosis. Otolaryngol Head Neck Surg. 2002; 127:22-31.

4-Charfi S, Ayadi L, Makni S. et al. Mucormycose rhinocérébrale : étude anatomoclinique de sept cas. J Mycol Med. 2008 ; $18: 46-52$.

5 -Zeddinia A, Meknia A, Ferchichia L. et al. Mucormycose rhinocérébrale : à propos d'un cas. Med Mal Infect. $2006 ; 36: 517-519$.

6- Cherif R, Ben Ali A, Gastli M, Chaker E, Daoud A. Zygomycose (mucormycose) rhino-orbito-cérébrale avec diabète insipide. Ann Fr Anesth Reanim. 1995; 14:41-44.

7- Belhadj SE, Daoud A, Gastli M. et al. Mucormycose rhino-orbitaire et diabète. À propos de six cas tunisiens. J Mycol Med. 1997; 7:159-61.

8- Chadli-Chaieb M, Bchir A, Fathallah-Mili A. et al. La mucormycose chez le diabétique. Presse Med 2005; 34:218-22.

9- Mnif N, Hmaied E, Oueslati S. et al. L'imagerie dans la mucormycose rhinocérébrale. J Radiol 2005; 86:1017-20.
10- Leitner C, Hoffmann J, Zerfowski M, Reinert S. Mucormycosis: necrotizing soft tissue lesion of the face. J Oral Maxillofac Surg 2003; 61(11):1354-8.

11- Slama A, Saghrouni F, Gaied-Meksi S. et al. Mucormycose post-traumatique de la face : à propos d'un cas. J Mycol Med. 2008 ;18:111-115.

12- Carton S, Garson S, Benhaim T. et al Mucormycose cutanée primaire : à propos d'un cas. Ann Chir Plast Esthet. 2008 ; 53:368-371.

13- Garbino J, Uckay I, Amini K, Puppo M, Richter M, Lew D. Absidia post-traumatic infection: successful treatment with posaconazole. J Infect 2005; 51:135-

14- Herbrecht R, Chabasse D. Zygomycoses : généralités et mucormycoses. Encycl. Med. Chir. (Elsevier, Paris). Techniques chirurgicales-Chir plast recons et esthétique, 8-614-B-10.

15- Guevara N, Roy D, Dutruc-Rosset C, Santini J, Hofman P, Castillo L. Mucormycose - diagnostic précoce et traitement. Rev Laryngol Otol Rhinol (Bord). 2004; 125:127-31.

16- Charfeddine I, Dhouib H, Hammami B. et al Mucormycose rhinosinusienne. A propos d'un cas. J. Tun ORL. $2005 ; 14: 42-45$.

17- Yohai RA, Bullock JD, Aziz AA, Markert RJ. Survival factors in rhino-orbitalcerebral mucormycosis. Surv Ophtalmol 1994; 39:3-22. 